\title{
Ventajas del uso del biodiesel B10 (Ricinus communis) sobre el Diésel fósil en la protección del medio ambiente
}

\author{
Advantages of the use of biodiesel B10 (Ricinus communis) on Diesel fossil in the protection \\ of the environment

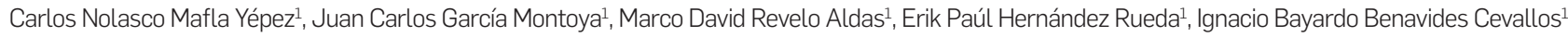

DOI. 10.21931/RB/2018.03.02.4

Abstract: The presence of soot in the environment has been considerably increasing lately according to the World Health Organization. One of the causes of this issue is due to the diesel engines. The study focuses on evaluating the presence of soot on an engine by using fuels such as Ricinus communis biodiesel and fossil diesel. The experiment was carried out using a diesel engine test bench Mazda BT50 with CRDi system. In order to run the tests, a BrainBee opacimeter was used. The opacimeter was connected into a probe, which is placed at the end of the car exhaust pipe. The experiment consists of testing the engine for several accelerations such as 1200, 1500, 2000, 2500, 3000 revolutions per minute (RPM) using Ricinus communis biodiésel and fossil diesel. Once all the accelerations are completed, the opacity difference was obtained to make a comparative analysis from the highest to the lowest opacity, resulting as the average percentage of opacity, which is the result that should be followed according to the established norms. It was observed $80 \%$ of reduction in diesel engine opacity with the use of Ricinus communis biodiésel comparing to fossil diesel.

Keywords: Biodiesel, Mazda BT 50 CRDi, Opacimeter, Soot, RPM.

Resumen: La presencia de el hollín en el ambiente ha aumentado considerablemente según la Organización Mundial de la Salud. Uno de los causantes de esta problemática son los motores diésel. El objetivo del presente estudio es evaluar el hollín que se emite de un motor diésel utilizando biodiésel de Ricino y diésel. Las pruebas se realizaron en un banco de motores diésel Mazda BT50-CRDi. Para realizar las mediciones se utilizó un opacímetro de marca BrainBee. El opacímetro está conectado a una sonda que se coloca en el tubo de escape, la cual transporta los gases al equipo. La prueba consiste en someter al motor a varias aceleraciones como son 1200, 1500, 2000, 2500, 3000 revoluciones por minuto (RPM) con el biodiésel y con el diésel fósil. Una vez completado todas las aceleraciones, se obtiene la diferencia de opacidad, básicamente sirve para realizar un análisis comparativo de la opacidad mayor con la menor, resultando un promedio porcentual de opacidad, que es el que se debe regular según las normas establecidas. Se notó una reducción del 80\% de opacidad en motores diésel con el uso de biodiésel a partir de aceite de Ricino en comparación al diésel fósil.

Palabras clave: Biodiésel, Mazda BT50 CRDi, Opacimetro, Hollín, RPM.

\section{Introducción}

Las emisiones de gases de efecto invernadero (GEI) (NOx, $\mathrm{CO}_{2}, \mathrm{CO}, \mathrm{HC}$ ) son los causantes de provocar el calentamiento global, el deterioro del ambiente y la salud de los seres humanos ${ }^{1}$. Se puede notar que con el pasar del tiempo existe una mayor concentración en la atmosfera terrestre por la globalización y las actividades antrópicas². Según la Organización (2014) Meteorológica Mundial, la concentración del $\mathrm{CO}_{2}$ se ha elevado en un 41\% en los últimos 10 años.

El motor diésel juega un papel importante en la producción industrial moderna y ofrece el funcionamiento eficiente, económico y fiable que se han reconocido en el transcurso de los años. Recientemente, China uno de los países más contaminados del mundo ha planteado una exigencia de emisiones más severa para el motor diésel, y está alineándose con el estándar Euro IV, PM y NOx en las emisiones generadas por el motor diésel ${ }^{3}$.

Es así como el ambiente se ha visto afectado por estas emisiones que son producto principalmente de la combustión de combustibles fósiles en motores a gasolina y diésel ${ }^{4}$. Se conoce que el parque automotor de vehículos con motor a gasolina y diésel va en aumento, siendo estos últimos los responsables de la emisión de partículas de hollín al ambiente ${ }^{5}$. Por otro lado, a parte de los gases de efecto invernadero (GEI) existen otras afectaciones ambientales producto de la combustión interna de motores que causan en la salud de los seres vivos problemas respiratorios y de piel.

El hollín, la ceniza y el polvo son partículas relativamente pesadas que se depositan en el suelo, y el aire las limpia rápidamente $^{6}$. Sin embargo, cerca de su fuente de origen constituyen con frecuencia un peligro para la salud, ya que pueden causar enfermedades respiratorias crónicas tales como el enfisema y el cáncer? ${ }^{7}$. Las emisiones de los motores de combustión afectan seriamente al medio ambiente y la salud humana. El calentamiento global está aumentando debido a los gases de efecto invernadero, provocado por los mismos.

Existen combustibles alternativos como el biodiésel de carbón neutro, que se debe tomar en cuenta como mecanismo para reducir las emisiones de gases contaminantes que se producen en la combustión ${ }^{8}$. Aún existe debate sobre el uso de biodiésel que provocan dificultades al momento de su uso por las mezclas que se utilizan, y dependiendo de dichas mezclas

\footnotetext{
${ }^{1}$ Universidad Técnica del Norte, Carrera de Ingeniería Automotriz, Grupo de investigación BICER, Ciudadela Universitaria Av. 17 de julio 5-21 y General José
} María Córdova sector El Olivo, CP. EC100150 Ibarra-Ecuador 
Las respectivas modificaciones del motor ${ }^{9}$, siendo el objetivo de este estudio, es evaluar la presencia de hollín en un motor a diésel utilizando biodiésel de Ricino y diésel fósil bajo diferentes regímenes del motor.

Enlaactualidad existen varios procesos detransesterificación para la producción de biodiésel. Los aceites vegetales tienen propiedades que permiten poder manipular su densidad y viscosidad, siendo adecuados para la producción de biodiése ${ }^{10}$. La transesterificación es el proceso químico de aceites vegetales que se utiliza alcohol con la combinación de un catalizador que puede ser hidróxido de potasio o hidróxido de sodio, para romper las moléculas del aceite vegetal en ésteres de etilo o metilo, obteniendo glicerina como subproducto y así poder separar el aceite y la glicérica ${ }^{11}$. En la producción de biodiésel, se realiza el proceso de evaporación del agua, donde se somete el combustible a una temperatura de $100^{\circ} \mathrm{C}$ para evaporar el agua utilizada en el lavado y poder obtener el Biodiésel que cumplan con las normas ASTM D 746712,13. Luego se mezcla el biodiésel preparado con diésel fósil en la concentración de (B10) para la fabricación de mezclas de biodiésel a ser utilizado en el motor diésel CRDi para la realización de pruebas de motores.

Al utilizar biodiésel se logra disminuir las emisiones de CO y CO2 presentes en la combustión debido a que una molécula de biodiésel contiene entre 12 y 18 carbones, y una molécula de diésel puede contener hasta 20 carbones $^{14,15}$. En el motor diésel la combustión se origina al comprimir el aire a presiones y temperaturas elevadas. Cuando el inyector entrega el diésel a la cámara, este ocasiona una auto ignición instantánea, al no poder oxidar toda la mezcla, el motor emite al ambiente hollín ${ }^{16}$, en altas cantidades este gas puede ser nocivo para los humanos ${ }^{17}$.

En el Ecuador el parámetro de medición se detalla en el Instituto Ecuatoriano de Normalización en la norma técnica NTE INEN 2 202:2000, donde manifiesta que "Los límites máximos de opacidad de emisiones para fuentes móviles con motor de diésel es: a partir del año 2000 en adelante, el 50\% de opacidad y los modelos posteriores al año 2000 es el 60\% de opacidad"18.

A nivel internacional la normativa de opacidad para vehículos con motores diésel indica que no debe superar el $40 \%$ del coeficiente de absorción de luz $\left(4 \mathrm{~m}^{-1}\right)^{19}$. El sistema de inyección de combustible en un motor diésel de inyección directa es alcanzar un alto grado de atomización para mejorar la penetración de combustible con la intención de utilizar la máxima cantidad de aire y para fomentar la evaporación en un corto tiempo y alcanzar una mayor eficiencia de combustión ${ }^{20}$.

\section{Materiales y métodos}

Para analizar la opacidad de los gases de escape se utilizó un banco de pruebas experimentales de motores diésel marca Mazda BT50 año 2013, el cual tiene una potencia de 157,30 HP a 3500 RPM y un torque de 363 Nm a 1800 RPM, 4 cilindros con un sistema de inyección directa electrónica CRDi (Common Rail Diésel Injection), cabe recalcar que para la experimentación no se hizo modificaciones al motor.

Para el estudio de opacidad de gases de escape en el motor mencionado se utilizó un opacímetro marca Brain Bee, modelo Opa 100, que permite la medición en base al principio de reducción de la intensidad de la luz que se utiliza para determinar el porcentaje de opacidad, que es la unidad de medición que determina el grado de opacidad de las emisiones de escape de una fuente móvil a diésel ${ }^{21}$.

Los combustibles que se utilizaron son el biodiésel procedente del aceite de ricino conocido como aceite de higuerilla y el diésel fósil del mercado. Para el caso del biodiésel se realizó una mezcla de B10 (10\% biodiésel y 90\% diésel fósil).

Las pruebas con los combustibles se desarrollaron en el mismo motor, tomando precauciones como la limpieza del sistema de inyección al momento de realizar cada una de las pruebas, con el fin de tener condiciones óptimas para la experimentación.

Las mediciones se realizaron cuando el motor llego a una temperatura de $80^{\circ} \mathrm{C}$, dicha temperatura se mide al aceite del motor, por medio de un termómetro que se conecta al opacímetro. Cuando se cumple esta condición, se procede a preparar el opacímetro con una función automática. Luego, se realiza una prueba para comprobar que la sonda no tenga fugas, estado que se denomina auto cero. Concluyendo con estos procedimientos se introduce la sonda al tubo de escape y se realiza las pruebas en diferentes revoluciones del motor ${ }^{22}$.

El opacímetro cuenta con valores de medición que van desde 0 a 99,9\% de opacidad, que consiste en evaluar la cantidad de hollín que pasa por un rayo de luz, y así conocer el porcentaje de opacidad que emite el motor diésel, de igual manera se puede testar con el coeficiente de absorción luminosa por metro $\left(\mathrm{K} \mathrm{a} \mathrm{m}^{-1}\right)$. Para tener una correcta medición, es necesario que los gases de escape cuenten con una temperatura mínima de $20{ }^{\circ} \mathrm{C}$ y una máxima de $400{ }^{\circ} \mathrm{C}$.

Para realizar la prueba de opacidad con los diferentes combustibles, se realizan 7 repeticiones a diferentes revoluciones como son 1200 RPM, 1500 RPM, 2000 RPM, 2500 RPM y 3000 RPM, esto para simular el comportamiento de diferentes estados del motor. Una vez que se obtiene los datos de opacidad en cada aceleración, se tiene la diferencia de opacidad que es la comparación de la unidad mayor con la menor, y para obtener el porcentaje de opacidad se calcula la media de todos los datos obtenidos.

\section{Resultados}

Como se observa en la figura 1, el porcentaje de opacidad aumenta cuando las RPM del motor se incrementan. Esto se debe a que a mayores revoluciones existe un mayor consumo de combustible y por ende una mayor cantidad de combustible no quemado, ocasionando así mayor cantidad de hollín. La norma NTE INEN 2 202:2000, exhibe que para este tipo de vehículo el límite de opacidad es de 50\%.

La conducción eficiente pasa siempre por mantener una marcha lo más regular posible aprovechando la inercia que el vehículo ha adquirido ${ }^{23}$. Una velocidad de crucero muy recomendable a este respecto se mantiene normalmente alrededor de los 2000 RPM teniendo engranada, por supuesto, la última velocidad hacia adelante. En este punto se suele dar el hecho de mantener el par máximo en el mínimo número de revoluciones, zona en la que se encuentra el menor consumo de combustible posible ${ }^{24}$.

Analizando la línea de tendencia se obtienen las ecuaciones para calcular la opacidad de motores a diésel y biodiésel según las RPM del motor, donde la opacidad a bajas revoluciones del motor (ralentí) sería de 0,5\% y a altas revoluciones (corte de inyección) 2,8\%.

\section{Discusión}

En la figura 1 se observa los resultados de opacidad utilizando los combustibles diésel fósil y biodiésel. Se tomaron en cuenta los mismos parámetros para ambos combustibles, 
Figure 1. Comparación del porcentaje de opacidad diésel y B10
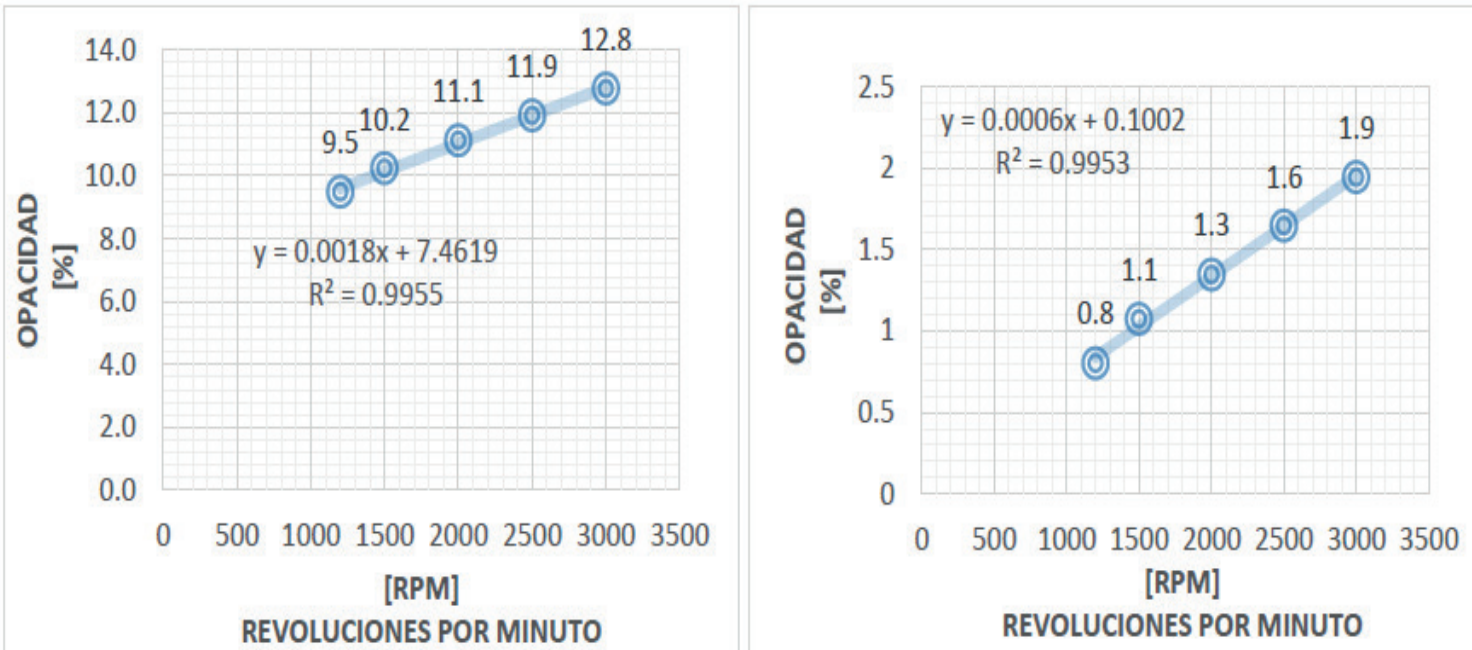

$$
O p a_{D F}=[0,0018(R P M)+7,4619]
$$

Donde.

Opa: Opacidad Diésel Fósil

RPM: Revoluciones del motor

$$
O p_{B 10}=[0,0006 \times(R P M)+0,1002]
$$

Donde,

Op B10: Opacidad Biodiésel

RPM: Revoluciones motor

evidenciando que para el diésel fósil el porcentaje de opacidad aumenta en rangos considerables en un $11,1 \%$ (Media) a diferencia del B10 en un 1,34\% (Media). Tomando en cuenta que el banco de pruebas es electrónico, este motor está por debajo de los límites de opacidad exigidos por las normas nacionales.

Analizando los resultados se tiene que a 1200 RPM se obtiene 9,5\% de opacidad y a 3000 RPM 12,8\% de opacidad. Considerando todos los datos se calcula un promedio del porcentaje de opacidad de 11,1\% para diésel fósil. En lo que respecta a la línea de tendencia para el combustible diésel fósil, se demuestra como lineal por lo que la opacidad es proporcional a las RPM del motor, como se observa la ecuación 1 para diésel fósil y la ecuación 2 para B10.

Comparando las líneas tendenciales entre los combustibles B10 y diésel fósil una determinada diferencia en el porcentaje de opacidad, que emiten los vehículos con motores diésel. Analizando el incremento de opacidad en cada RPM es mayor en comparación con B10 de la misma manera al aumentar las RPM se eleva el porcentaje de opacidad, pero se puede observar la diferencia en la línea de tendencia donde en las mismas RPM el aumento de opacidad es menor. Se observa que al aumentar 500 RPM el motor con el uso de diésel fósil aumenta 9 puntos de opacidad en cada prueba, y con el uso de B10 aumenta 3 puntos de opacidad disminuyendo un 80\% las emisiones de hollín en el motor. Analizando la opacidad a mayores revoluciones donde su presencia es mayor se puede concluir que con el uso de B10 se disminuye un $85 \%$ de opacidad a 3000 RPM. Estos datos se evidencian en base a la tendencia que se genera.

Analizando los resultados obtenidos se puede concluir que con el uso de biodiésel de aceite de higuerilla (Ricino communis), disminuye considerablemente la presencia de partículas de hollín en gases de escape de los motores diésel.

Tomando en cuenta los parámetros cuando el motor es más eficiente a 2500 RPM, se determinó que la opacidad con B10 es 1,6\%, resaltando así que la emisión de hollín es baja comparada con el diésel fósil, de esta manera se puede decir que la mezcla B10 disminuye considerablemente en 80 veces 
la contaminación de hollín en un motor a diésel con esas características.

\section{Conclusiones}

Analizando los datos obtenidos en las pruebas con uso de B10 y diésel fósil se llegó a la conclusión que con el B10 se puede lograr una disminución del $80 \%$ de opacidad en los motores a diésel con esas características, esto se debe a la disminución de partículas de carbón en el biodiésel y esto contribuye a que se oxide mejor la mezcla combustible aire.

A mayores revoluciones del motor mayor consumo de combustible y por ende acrecienta la formación de hollín, tomando en cuenta las condiciones óptimas de funcionamiento del motor que está en el rango de 2500 RPM, se concluye que con el uso de B10 se disminuye 10 veces la presencia de hollín.

Aplicando las ecuaciones obtenidas en el estudio tanto para B10 como diésel fósil se puede obtener la opacidad según las revoluciones a las que trabaje el motor.

Como se puedo apreciar los motores diésel electrónicos CRDi cumplen con la normativa establecida en el Ecuador, ya que no superan los límites de opacidad con ninguno de los combustibles utilizados, pero la presencia de menos cantidad de hollín en el ambiente contribuye a la conservación del mismo.

Según la Organización Mundial de Salud el hollín emitido por los vehículos diésel son los una de las causas de enfermedades cancerígenas en los pulmones de los humanos, donde las partículas más pequeñas de hollín son las más críticas, pero con la utilización de biodiésel se podría mitigar este problema.

\section{Referencias bibliográficas}

1. Zambrano G, Rossi C. y Hernandez J.: "Emisión de gases en vehículos experimentales diesel-biodiesel". Universidad del Valle Guatemala, Vol. 12, No. 5 (2014) 64-67.

2. Udiel D. y Solano M.: "Contaminación por emisiones vehiculares sector residencial linda vista sur, distrito número dos de managua, Universidad Nacional Autonoma de Nicaragua, Managua, 2013.

3. Zhen Z. y Jing L.: "Effects of Exhaust Post-treatment Technology on Diesel Engine Emissions". Proceedings 2011 International Conference on Transportation, Mechanical, and Electrical Engineering, (2011) 1804-1807.

4. Jiaqiang E., Minhhieu P., Zhao D., Yuanwang D., DucHieu L., Wei Z., Teng L., Qingguo P. y Zhiqing Z.: "Effect of different technologies on combustion and emissions of the diesel engine fueled with biodiesel: A review". Renewable and Sustainable Energy Reviews, Vol. 80, (2017) 620-647.

5. Delgado A., Chaparro W. y Silva J.: "Influencia del porcentaje de mezcla del aceite de higuerilla en la obtención de combustible alternativo para motores diesel". Revista Facultad Ingeniería Universidad Antioquia, Vol. 5, (2011) 45-52.

6. J. Azjargal, «The Comparison of Combustion Characteristic and Performances of Diesel Engine Fueled with Biodiesel and Diesel Blends". International Forum on Strategic Technology, Vol. 9 , (2014) 313-316.

7. Organización Mundial de la Salud.: "Calidad del aire ambiente (exterior) y salud". OMS, Ginebra, 2016.

8. Honig V., Strouhal J. y Linhart Z.: "Biodiesel from waste animal fat: efficient fuel of the future". International Advances in Economic Research, Vol. 25, (2016) 465-477.

9. Imtenan S., Varman M., Masju H., Kalam M., Sajjad H., Arbab M. y Rizwanul I.: "Impact of low temperature combustion attaining strategies on diesel engine emissions for diesel and biodiesels:
A review". Energy Conversion and Management, Vol. 80, (2014) 329-356.

10. Aalam C., Saravanan G. y Kannan M.: "Experimental investigations on a CRDI system assisted diesel engine fuelled with aluminium oxide nanoparticles blended biodiesel". Alexandria Engineering Journal, Vol. 70, No. 3, (2015) 351-358.

11. Rojas A., Girón E. y Torres H.: "Variables de operación en el proceso de transesterificación de aceites vegetales: una revisión catálisis química". Ingeniería e Investigaión, Vol. 30, No. 1, (2009) 17-22.

12. Senila L., Miclean M., Cadar O., Senila M., Kovacs M. y Hoaghia A.: "Validation of a method for determination of free glycerol in biodiesel". Studia Universitatis Babes-Bolyai. Chemia., Vol. 15, (2016) 345-348.

13. Maksimuk Y., Buglak A., Kruk V. y Antonova Z.: "Rheological properties of residual fuel oil containing fuel blends with bioadditives. Part 1. Rapeseed oil based bioadditives". Chemistry and Technology of Fuels and Oils, Vol. 9, (2013) 108-115.

14. Amaris J., Manrique D. y Jaramillo J.: "Biocombustibles líquidos en Colombia y su impacto en motores de combustión interna". El Reventón Energético, (2015) 23-34.

15. Karlsson H., Ahlgren S., Sandgren M., Passoth V., Wallberg O. y Hansson A.: "A systems analysis of biodiesel production from wheat straw using oleaginous yeast: process design, mass and energy balances". Biotechnology for Biofuels, Vol. 34 (2016) 356-378.

16. Agudelo J., Agudelo A. y Pérez J.: "Análisis energético y exergético de un motor diesel de automoción operando en diferentes altitudes". Revista Facultad Ingeniería Universidad Antioquia, Vol. 8, (2009) 45-54.

17. Lalvani J., Kirubhakaran K., Parthasarathy M. y Sabarish R.: "Performance Characteristic and Emission Analysis of a Single Cylinder Diesel Engine Operated on Blends of Diesel and Easte Cooking Oil". 2013 International Conference on Energy Efficient Technologies for Sustainability, (2013) 781-785.

18. Instituto Ecuatoriano de Normalización, Gestión ambiental, Aire, Vehículos automotores.: "Determinación de la opacidad de emisiones de escape de motores de diesel mediante la prueba estática. Método de aceleración libre". Quito, INEN, 2000.

19. Asociación americana de testeo de materiales.: "Densidad del humo de gases de escape de la combustión". ASTM internacional, EEUU, 2013.

20.Syed A., Saravanan C. y Anand P.: "Impacto de la alta presión de inyección de combustible de las características del motor CRDI diesel alimentado por mahua mezcla de ésteres de metilo". Ingeniería Térmica Aplicada, Vol. 106, (2016) 702-711.

21. Brain Bee.: "OPA 100 Opacimetro para motores diésel". Parma Italia: Brain Bee S.p.A, 2010.

22. Globaltech.: "Opacimetro Brain Bee Opa100". (2016). [En línea]. Available: http://globaltechla.com/equipos-de-taller/34-opacimetro-opa-100.html\#.WHk9mFN97IU.

23. Institution of Mechanical Engineers, "Internal Combustion Engines: Improving Performance, Fuel Economy and Emission". London: Woodheat Publishing, 2011.

24.Castro V.: "El motor diésel en el automóvil". CEAC, Barcelona, 2000.

Recibido: 9 noviembre 2017

Aprobado: 2 enero 2018 